\title{
Approximation by Cheney-Sharma Chlodovsky operators
}

\author{
Dilek Söylemez $^{* 1}$ (D), Fatma Taşdelen ${ }^{2}$ \\ ${ }^{1}$ Ankara University, Elmadag Vocational School, Department of Computer Pogramming, 06780, Ankara, \\ Turkey \\ ${ }^{2}$ Ankara University, Faculty of Science, Department of Mathematics, 06100 Tandogan, Ankara, Turkey
}

\begin{abstract}
The main purpose of this paper is to construct Cheney-Sharma Chlodovsky operators. We study approximation properties of the new operators with the help of weighted Korovkintype theorem and universal Korovkin-type theorem. We also give the rate of convergence by means of the modulus of continuity. Furthermore, we give $A$-statistical convergence property of these operators.
\end{abstract}

Mathematics Subject Classification (2010). 41A36, 40A35

Keywords. Cheney-Sharma Chlodovsky operators, weighted approximation, rate of approximation, $A$-statistical approximation

\section{Introduction}

Approximation theory is concerned with how functions can best be approximated with simpler functions. Since Korovkin theorem was obtained, the studies of the linear methods of approximation given by sequences of positive and linear operators have become an important area in approximation theory. The study of the statistical convergence in approximation theory for sequences of linear positive operators was attempted by Gadjiev and Orhan [23]. They proved a Korovkin type theorem by considering statistical convergence instead of ordinary convergence. Later, many authors gave several approximation results via summability methods, for example, we refer the readers to $[1,2,4,16,28,29,31,32,34]$.

In 1932, Chlodovsky [14], introduced the classical Bernstein-Chlodovsky polynomials as a generalization of Bernstein polynomials on unbounded set.

For every $n \in \mathbb{N}, f \in C[0, \infty)$ and $x \in[0, \infty)$, these polynomials $C_{n}: C[0, \infty) \rightarrow C[0, \infty)$ defined by

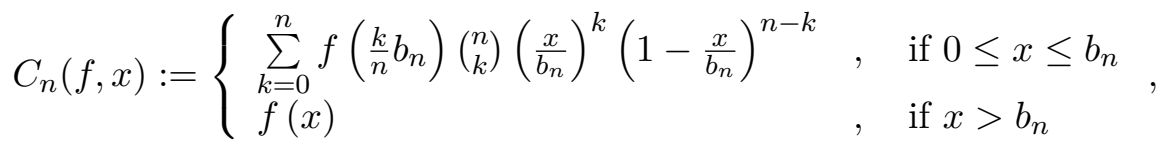

where $0 \leq x \leq b_{n}$ and $\left\{b_{n}\right\}$ is a positive sequence with the properties;

$\lim _{n \rightarrow \infty} b_{n}=\infty, \lim _{n \rightarrow \infty} b_{n} / n=0$.

\footnotetext{
*Corresponding Author.

Email addresses: dsoylemez@ankara.edu.tr (D. Söylemez), tasdelen@science.ankara.edu.tr (F. Taşdelen)

Received: 10.10.2018; Accepted: 06.01.2019
} 
Recently some authors have studied some Chlodovsky type polynomials which may be found in $[6,12,24-27,30]$.

It is known that the Abel-Jensen equalities are given by the following formulas (see [5], p.326)

$$
(u+v+n \beta)^{n-1}=\sum_{k=0}^{n}\left(\begin{array}{l}
n \\
k
\end{array}\right) u(u+k \beta)^{k-1} v[v+(n-k) \beta]^{n-k-1}
$$

and

$$
(u+v)(u+v+m \beta)^{m-1}=\sum_{k=0}^{m}\left(\begin{array}{c}
m \\
k
\end{array}\right) u(u+k \beta)^{k-1} v[v+(m-k) \beta]^{m-k-1},
$$

where $u, v$ and $\beta \in \mathbb{R}$. By means of these equalities Cheney-Sharma [13] introduced the following Bernstein type operators for $f \in C[0,1], x \in[0,1]$ and $n \in \mathbb{N}$

$$
\begin{aligned}
Q_{n}(f ; x)= & (1+n \beta)^{1-n} \sum_{k=0}^{n} f\left(\frac{k}{n}\right)\left(\begin{array}{l}
n \\
k
\end{array}\right) x(x+k \beta)^{k-1} \\
& \times[1-x+(n-k) \beta]^{n-k}
\end{aligned}
$$

and

$$
\begin{aligned}
G_{n}(f ; x)= & (1+n \beta)^{1-n} \sum_{k=0}^{n} f\left(\frac{k}{n}\right)\left(\begin{array}{l}
n \\
k
\end{array}\right) x(x+k \beta)^{k-1} \\
& \times(1-x)[1-x+(n-k) \beta]^{n-k-1}
\end{aligned}
$$

where $\beta$ is a nonnegative real parameter. It is obvious that for $\beta=0$ these operators reduce to the classical Bernstein operators. Cheney-Sharma proved that if $n \beta_{n} \rightarrow \infty$ as $n \rightarrow \infty$, then for $f \in C[0,1]$, these operators uniformly convergence to $f$ on $[0,1]$. In [7], the authors showed that Cheney-Sharma operators preserve the Lipschitz constant and order of a Lipschitz continuous function as well as the properties of the function of modulus of continuity. They also gave a result of $G_{n}(f ; x)$ under the convexity of $f$. Kantorovich type generalization of the Cheney-Sharma operators was studied in [33]. For these operators, we refer the readers to $[3,8,15,35,36]$.

In the present paper, we construct the Chlodovsky-type generalization of the CheneySharma operators is given by (1.4) and we prove that the weighted uniform convergence of $G_{n}^{*} f$ to $f$. We also give approximation results using universal Korovkin-type theorem and obtain rate of approximation in terms of the usual modulus of continuity. Finally, we study A-statistical convergence behaviours of new positive linear operators expressed as below.

We introduce the Cheney-Sharma Chlodovsky operators as

$$
\begin{aligned}
G_{n}^{*}(f ; x)= & (1+n \beta)^{1-n} \sum_{k=0}^{n} f\left(\frac{k}{n} b_{n}\right)\left(\begin{array}{l}
n \\
k
\end{array}\right) \frac{x}{b_{n}}\left(\frac{x}{b_{n}}+k \beta\right)^{k-1} \\
& \times\left(1-\frac{x}{b_{n}}\right)\left[1-\frac{x}{b_{n}}+(n-k) \beta\right]^{n-k-1}
\end{aligned}
$$

for $0 \leq x \leq b_{n}$ and $f(x)$ for $x>b_{n}$. Here $\left\{b_{n}\right\}$ is a positive sequence with the properties; $\lim _{n \rightarrow \infty} b_{n}=\infty, \lim _{n \rightarrow \infty} b_{n} / n=0$. If we take $b_{n}=1$, then we obtain the Cheney-Sharma operators (1.4).

Now, let us recall the concept of $A$-statistical convergence. Let $A=\left(a_{n k}\right)$ be a summability matrix and let $x=\left(x_{k}\right)$ be a sequence.

If the series

$$
(A x)_{n}:=\sum_{k} a_{n k} x_{k}
$$


is convergent for each $n$ then, we say that $A x:=\left\{(A x)_{n}\right\}$ is the A-transformation of $x$. If the sequence $A x$ converges to a number $L$ then, we say that $x$ is $A$-summable to $L$. A summability matrix $A$ is said to be regular if $\lim _{n}(A x)_{n}=L$ whenever $\lim _{k} x_{k}=L$ [9].

Let $A=\left(a_{n k}\right)$ be a nonnegative regular summability matrix and let $K$ be a subset of positive integer. Then $K$ is said to have $A$-density $\delta_{A}(K)$ if the limit

$$
\delta_{A}(K):=\lim _{n} \sum_{k \in K} a_{n k}
$$

exists $[10,11,19]$. The sequence $x=\left(x_{k}\right)$ is said to be $A$-statistically convergent to real number $\alpha$ if for any $\varepsilon>0$

$$
\lim _{k} \sum_{k:\left|x_{k}-\alpha\right| \geq \varepsilon} a_{n k}=0
$$

In this case, we write $s t_{A}-\lim x=\alpha[18,20]$. Note that $x$ is $A$-statistically convergent to $\alpha$ if and only if for any $\varepsilon>0, \delta_{A}\left(K_{\varepsilon}\right)=0$, where $K_{\varepsilon}:=\left\{k \in \mathbb{N}:\left|x_{k}-\alpha\right| \geq \varepsilon\right\}$. If $A$ is the identity matrix $I$, then $I$-statistical convergence reduces to ordinary convergence, and, if $A=C_{1}$, the Cesáro matrix of order one, then it coincides with statistical convergence.

Throughout the paper, we will consider the following class of functions. Let $\rho(x)=$ $1+x^{2}$,

$$
B_{\rho}\left(\mathbb{R}^{+}\right)=\left\{f: \mathbb{R}^{+} \rightarrow \mathbb{R},|f(x)| \leq M_{f} \rho(x), x \geq 0\right\},
$$

where $M_{f}$ is a constant depending on $f$.

$$
\begin{gathered}
C_{\rho}\left(\mathbb{R}^{+}\right)=\left\{f \in B_{\rho}\left(\mathbb{R}^{+}\right) ; f \text { is continuous on } \mathbb{R}^{+}\right\}, \\
C_{\rho}^{k}\left(\mathbb{R}^{+}\right)=\left\{f \in C_{\rho}\left(\mathbb{R}^{+}\right) ; \lim _{x \rightarrow \infty} \frac{f(x)}{\rho(x)}=k_{f}\right\},
\end{gathered}
$$

where $k_{f}$ is a constant depending on $f$.

It is obvious that $C_{\rho}^{k}\left(\mathbb{R}^{+}\right) \subset C_{\rho}\left(\mathbb{R}^{+}\right) \subset B_{\rho}\left(\mathbb{R}^{+}\right)$. The space $B_{\rho}\left(\mathbb{R}^{+}\right)$is a normed linear space with the norm $\|f\|_{\rho}=\sup _{x \in \mathbb{R}^{+}} \frac{|f(x)|}{\rho(x)}$.

\section{Approximation by $\left(G_{n}^{*}\right)$}

Korovkin theorem was extended to unbounded intervals and a weighted Korovkin type theorem in a subspace of continuous functions on the real axis $\mathbb{R}$ was proved in $[21,22]$. Let us recall the weighted form of the Korovkin Theorem $([21,22])$.

Lemma 2.1. The positive linear operators $L_{n}, n \geq 1$, act from $C_{\rho}\left(\mathbb{R}^{+}\right)$to $B_{\rho}\left(\mathbb{R}^{+}\right)$if and only if the inequality

$$
\left|L_{n}(\rho ; x)\right| \leq K_{n} \rho(x), x \geq 0
$$

holds, where $K_{n}$ is a positive constant.

Theorem 2.2. Let the sequence of linear positive operators $\left(L_{n}\right)_{n \geq 1}$ acting from $C_{\rho}\left(\mathbb{R}^{+}\right)$ to $B_{\rho}\left(\mathbb{R}^{+}\right)$satisfy the three conditions

$$
\lim _{n \rightarrow \infty}\left\|L_{n}\left(t^{v} ; x\right)-x^{v}\right\|_{\rho}=0, v=0,1,2 .
$$

Then for any function $f \in C_{\rho}^{k}\left(\mathbb{R}^{+}\right)$

$$
\lim _{n \rightarrow \infty}\left\|L_{n} f-f\right\|_{\rho}=0 .
$$

Lemma 2.3. Let $e_{i}(t)=t^{i}, i=0,1,2$. For the operators (1.5), we have

$$
\begin{aligned}
& G_{n}^{*}\left(e_{0} ; x\right)=1, \\
& G_{n}^{*}\left(e_{1} ; x\right)=x
\end{aligned}
$$


and

$$
\begin{aligned}
G_{n}^{*}\left(e_{2} ; x\right) & \leq x\left(x+2 b_{n} \beta\right)(1+n \beta) \\
& +x \frac{b_{n}}{n}(n \beta)^{2}(1+n \beta)+x\left(x+2 b_{n} \beta\right) n \beta \\
& +x \frac{b_{n}}{n}(n \beta)^{3}+x \frac{b_{n}}{n} .
\end{aligned}
$$

Proof. Firstly, we show that $G_{n}^{*}\left(e_{i} ; x\right)$ for $i=0$,

$G_{n}^{*}\left(e_{0} ; x\right)=(1+n \beta)^{1-n} \sum_{k=0}^{n}\left(\begin{array}{l}n \\ k\end{array}\right) \frac{x}{b_{n}}\left(\frac{x}{b_{n}}+k \beta\right)^{k-1}\left(1-\frac{x}{b_{n}}\right)\left[1-\frac{x}{b_{n}}+(n-k) \beta\right]^{n-k-1}$.

If we take $u=\frac{x}{b_{n}}, v=1-\frac{x}{b_{n}}$ in $(1.2)$, we get

$$
G_{n}^{*}\left(e_{0} ; x\right)=1
$$

Morever, taking $G_{n}^{*}\left(e_{i} ; x\right)$ for $i=1$, one can have

$$
\begin{aligned}
& G_{n}^{*}\left(e_{1} ; x\right)=(1+n \beta)^{1-n} \sum_{k=1}^{n}\left(\begin{array}{l}
n-1 \\
k-1
\end{array}\right) b_{n} \frac{x}{b_{n}}\left(\frac{x}{b_{n}}+k \beta\right)^{k-1} \\
& \times\left(1-\frac{x}{b_{n}}\right)\left[1-\frac{x}{b_{n}}+(n-k) \beta\right]^{n-k-1} .
\end{aligned}
$$

If we take $k \rightarrow k+1$ and considering the equality $\frac{x}{b_{n}}+\beta+k \beta=1+n \beta-\left[1-\frac{x}{b_{n}}+(n-k-1) \beta\right]$, we reach to

$$
\begin{aligned}
G_{n}^{*}\left(e_{1} ; x\right)= & (1+n \beta)^{1-n} b_{n} \sum_{k=0}^{n-1}\left(\begin{array}{c}
n-1 \\
k
\end{array}\right) \frac{x}{b_{n}}\left(\frac{x}{b_{n}}+\beta+k \beta\right)^{k-1}(1+n \beta) \\
& \times\left(1-\frac{x}{b_{n}}\right)\left[1-\frac{x}{b_{n}}+(n-k-1) \beta\right]^{n-k-2} \\
& -(1+n \beta)^{1-n} b_{n} \sum_{k=0}^{n-1}\left(\begin{array}{c}
n-1 \\
k
\end{array}\right) \frac{x}{b_{n}}\left(\frac{x}{b_{n}}+\beta+k \beta\right)^{k-1} \\
& \times\left(1-\frac{x}{b_{n}}\right)\left[1-\frac{x}{b_{n}}+(n-k-1) \beta\right]^{n-k-1} .
\end{aligned}
$$

In order to find the first sum, we replace in the identity (1.2), $u=\frac{x}{b_{n}}+\beta, v=1-\frac{x}{b_{n}}, n$ by $n-1$, we get

$$
\begin{gathered}
(1+\beta)(1+n \beta)^{n-2}=\left(\frac{x}{b_{n}}+\beta\right) \sum_{k=0}^{n-1}\left(\begin{array}{c}
n-1 \\
k
\end{array}\right)\left(\frac{x}{b_{n}}+\beta+k \beta\right)^{k-1} \\
\times\left(1-\frac{x}{b_{n}}\right)\left[1-\frac{x}{b_{n}}+(n-k-1) \beta\right]^{n-k-2} .
\end{gathered}
$$

If we use (2.8) in (2.7), we have

$$
\begin{aligned}
G_{n}^{*}\left(e_{1} ; x\right)= & (1+\beta) \frac{x b_{n}}{x+b_{n} \beta}-(1+n \beta)^{1-n} b_{n} \sum_{k=0}^{n-1}\left(\begin{array}{c}
n-1 \\
k
\end{array}\right) \frac{x}{b_{n}}\left(\frac{x}{b_{n}}+\beta+k \beta\right)^{k-1} \\
& \times\left(1-\frac{x}{b_{n}}\right)\left[1-\frac{x}{b_{n}}+(n-k-1) \beta\right]^{n-k-1} .
\end{aligned}
$$


On the other hand, if we take $u, v$ and $n$ by $\frac{x}{b_{n}}+\beta, 1-\frac{x}{b_{n}}$ and $n-1$, respectively in (1.1), we get

$$
\begin{aligned}
(1+n \beta)^{n-1}= & \sum_{k=0}^{n-1}\left(\begin{array}{c}
n-1 \\
k
\end{array}\right)\left(\frac{x}{b_{n}}+\beta\right)\left(\frac{x}{b_{n}}+\beta+k \beta\right)^{k-1} \\
& \times\left[1-\frac{x}{b_{n}}+(n-k-1) \beta\right]^{n-k-1}
\end{aligned}
$$

By considering (2.10) in (2.9), so we have

$$
G_{n}^{*}\left(e_{1} ; x\right)=x
$$

Finally, we estimate $G_{n}^{*}\left(e_{i} ; x\right)$ for $i=2$,

$$
\begin{aligned}
G_{n}^{*}\left(e_{2} ; x\right)= & (1+n \beta)^{1-n} \frac{n-1}{n}\left(b_{n}\right)^{2} \sum_{k=0}^{n-2}\left(\begin{array}{c}
n-2 \\
k
\end{array}\right) \frac{x}{b_{n}}\left(\frac{x}{b_{n}}+k \beta+2 \beta\right)^{k+1} \\
& \times\left(1-\frac{x}{b_{n}}\right)\left[1-\frac{x}{b_{n}}+(n-k-2) \beta\right]^{n-k-3} \\
& +(1+n \beta)^{1-n} \frac{b_{n}}{n} \sum_{k=1}^{n} \frac{k}{n} b_{n}\left(\begin{array}{c}
n \\
k
\end{array}\right) \frac{x}{b_{n}}\left(\frac{x}{b_{n}}+k \beta\right)^{k-1} \\
& \times\left(1-\frac{x}{b_{n}}\right)\left[1-\frac{x}{b_{n}}+(n-k) \beta\right]^{n-k-1} \\
= & (1+n \beta)^{1-n} \frac{n-1}{n}\left(b_{n}\right)^{2} \frac{x}{b_{n}} \sum_{k=0}^{n-2}\left(\begin{array}{c}
n-2 \\
k
\end{array}\right)\left(\frac{x}{b_{n}}+k \beta+2 \beta\right)^{k+1} \\
& \times\left[1-\frac{x}{b_{n}}+(n-k-2) \beta\right]^{n-k-2} \\
& -(1+n \beta)^{1-n} \frac{n-1}{n}\left(b_{n}\right)^{2} \frac{x}{b_{n}} \sum_{k=0}^{n-2}\left(\begin{array}{c}
n-2 \\
k
\end{array}\right) \frac{x}{b_{n}}\left(\frac{x}{b_{n}}+k \beta+2 \beta\right)^{k+1} \\
& \times(n-k-2) \beta\left[1-\frac{x}{b_{n}}+(n-k-2) \beta\right]^{n-k-3} \\
& +\frac{b_{n}}{n} G_{n}^{*}\left(e_{1} ; x\right) .
\end{aligned}
$$

In order to find an upper estimate for the $G_{n}^{*}\left(e_{2} ; x\right)$, we need to give the following equality, as in [13]

$$
L\left(j, n, \frac{x}{b_{n}}, 1-\frac{x}{b_{n}}\right):=\sum_{k=0}^{n}\left(\begin{array}{l}
n \\
k
\end{array}\right)\left(\frac{x}{b_{n}}+k \beta\right)^{k+j-1}\left(1-\frac{x}{b_{n}}+(n-k) \beta\right)^{n-k},
$$

where $n \in \mathbb{N}, 0 \leq x \leq b_{n}$ and $b_{n}$ is a sequence of positive numbers such that $\lim _{n \rightarrow \infty} b_{n}=$ $\infty, \lim _{n \rightarrow \infty} \frac{b_{n}}{n}=0$.

It is clear that the function $L\left(j, n, \frac{x}{b_{n}}, 1-\frac{x}{b_{n}}\right)$ satisfies the following reduction formula

$$
L\left(j, n, \frac{x}{b_{n}}, 1-\frac{x}{b_{n}}\right)=\frac{x}{b_{n}} L\left(j-1, n, \frac{x}{b_{n}}, 1-\frac{x}{b_{n}}\right)+n \beta L\left(j, n-1, \frac{x}{b_{n}}+\beta, 1-\frac{x}{b_{n}}\right) .
$$


Therefore from (2.12), we get

$$
\begin{aligned}
G_{n}^{*}\left(e_{2} ; x\right)= & \frac{x}{b_{n}}(1+n \beta)^{1-n} \frac{n-1}{n}\left(b_{n}\right)^{2} L\left(2, n-2, \frac{x}{b_{n}}+2 \beta, 1-\frac{x}{b_{n}}\right) \\
& -\frac{x}{b_{n}}(1+n \beta)^{1-n} \frac{n-1}{n}\left(b_{n}\right)^{2}(n-2) \beta L\left(2, n-3, \frac{x}{b_{n}}+2 \beta, 1-\frac{x}{b_{n}}+\beta\right) \\
& +\frac{b_{n}}{n} G_{n}^{*}\left(e_{1} ; x\right) \\
= & K_{1}(n, x)+K_{2}(n, x)+\frac{b_{n}}{n} x .
\end{aligned}
$$

From Lemma 1 in [13], we know that

$$
\begin{aligned}
L(2, n, x, y)= & \int_{0}^{\infty} e^{-s} d s \int_{0}^{\infty} e^{-u}\left[x(x+y+n \beta+s \beta+u \beta)^{n}\right. \\
& \left.+n \beta^{2} u(x+y+n \beta+s \beta+u \beta)^{n-1}\right] d u
\end{aligned}
$$

and so we have,

$$
\begin{aligned}
L(2, n & \left.-2, \frac{x}{b_{n}}+2 \beta, 1-\frac{x}{b_{n}}\right) \\
= & (1+n \beta)^{n-2} \int_{0}^{\infty} e^{-s} d s \int_{0}^{\infty} e^{-u}\left[\left(\frac{x}{b_{n}}+2 \beta\right)\left(1+\frac{s \beta+u \beta}{1+n \beta}\right)^{n-2}\right] d u \\
& +(1+n \beta)^{n-3} \int_{0}^{\infty} e^{-s} d s \int_{0}^{\infty} e^{-u}(n-2) \beta^{2} u\left(1+\frac{s \beta+u \beta}{1+n \beta}\right)^{n-3} d u \\
= & \alpha(n, x)+\beta(n, x) .
\end{aligned}
$$

Taking into account this inequality $(1+v)^{n-2} \leq \exp (n v)$, we have

$$
\begin{aligned}
\alpha(n, x) & \leq(1+n \beta)^{n-2} \int_{0}^{\infty} e^{-s} d s \int_{0}^{\infty} e^{-u}\left[\left(\frac{x}{b_{n}}+2 \beta\right) \exp \left(n\left(\frac{s \beta+u \beta}{1+n \beta}\right)\right)\right] d u \\
& =\left(\frac{x}{b_{n}}+2 \beta\right)(1+n \beta)^{n-2} \int_{0}^{\infty} e^{-s\left(\frac{1}{1+n \beta}\right)} d s \int_{0}^{\infty} e^{-u\left(\frac{1}{1+n \beta}\right)} d u \\
& =\left(\frac{x}{b_{n}}+2 \beta\right)(1+n \beta)^{n-2}(1+n \beta)^{2}
\end{aligned}
$$

and

$$
\begin{aligned}
\beta(n, x) & \leq(1+n \beta)^{n-3} \int_{0}^{\infty} e^{-s} d s \int_{0}^{\infty} e^{-u} u(n-2) \beta^{2} \exp \left(n\left(\frac{s \beta+u \beta}{1+n \beta}\right)\right) d u \\
& =(n-2) \beta^{2}(1+n \beta)^{n-3} \int_{0}^{\infty} e^{-s\left(\frac{1}{1+n \beta}\right)} d s \int_{0}^{\infty} u e^{-u\left(\frac{1}{1+n \beta}\right)} d u \\
& =(1+n \beta)^{n}(n-2) \beta^{2} .
\end{aligned}
$$


Taking (2.15) and (2.16) in (2.14), we get

$$
\begin{aligned}
K_{1}(n, x) \leq & \left(\frac{x}{b_{n}}+2 \beta\right) \frac{x}{b_{n}}(1+n \beta)^{1-n} \frac{n-1}{n}\left(b_{n}\right)^{2}(1+n \beta)^{n} \\
& +\frac{x}{b_{n}}(1+n \beta)^{1-n} \frac{n-1}{n}\left(b_{n}\right)^{2}(1+n \beta)^{n}(n-2) \beta^{2} \\
\leq & x\left(x+2 b_{n} \beta\right)(1+n \beta)+x \frac{b_{n}}{n}(n \beta)^{2}(1+n \beta) .
\end{aligned}
$$

On the other hand

$$
\begin{aligned}
K_{2}(n, x) \leq & \frac{x}{b_{n}}(1+n \beta)^{1-n} \frac{n-1}{n}\left(b_{n}\right)^{2}(n-2) \beta \\
& \times L\left(2, n-3, \frac{x}{b_{n}}+2 \beta, 1-\frac{x}{b_{n}}+\beta\right)
\end{aligned}
$$

and

$$
\begin{aligned}
L(2, n & \left.-3, \frac{x}{b_{n}}+2 \beta, 1-\frac{x}{b_{n}}+\beta\right) \\
= & \int_{0}^{\infty} e^{-s} d s \int_{0}^{\infty} e^{-u}\left(\frac{x}{b_{n}}+2 \beta\right)(1+n \beta+s \beta+u \beta)^{n-3} d u \\
& +\int_{0}^{\infty} e^{-s} d s \int_{0}^{\infty} e^{-u}(n-2) \beta^{2}(1+n \beta+s \beta+u \beta)^{n-4} d u \\
= & \gamma(n, x)+\xi(n, x) .
\end{aligned}
$$

Here

$$
\begin{aligned}
\gamma(n, x) & \leq(1+n \beta)^{n-3}\left(\frac{x}{b_{n}}+2 \beta\right) \int_{0}^{\infty} e^{-s} d s \int_{0}^{\infty} e^{-u} \exp \left(n\left(\frac{s \beta+u \beta}{1+n \beta}\right)\right) d u \\
& =(1+n \beta)^{n-3}\left(\frac{x}{b_{n}}+2 \beta\right) \int_{0}^{\infty} e^{-s\left(\frac{1}{1+n \beta}\right)} d s \int_{0}^{\infty} e^{-u\left(\frac{1}{1+n \beta}\right)} d u \\
& =(1+n \beta)^{n-3}\left(\frac{x}{b_{n}}+2 \beta\right)(1+n \beta)^{2}
\end{aligned}
$$

and

$$
\begin{aligned}
\xi(n, x) & =\int_{0}^{\infty} e^{-s} d s \int_{0}^{\infty} u e^{-u}(n-2) \beta^{2}(1+n \beta+s \beta+u \beta)^{n-4} d u \\
& \leq(1+n \beta)^{n-4}(n-2) \beta^{2}(1+n \beta)(1+n \beta)^{2} .
\end{aligned}
$$

So, taking $(2.19),(2.20)$ and $(2.21)$ in $(2.18)$, we have

$$
K_{2}(n, x) \leq x\left(x+2 b_{n} \beta\right) n \beta+x \frac{b_{n}}{n}(n \beta)^{3} .
$$

Hence, we can write

$$
\begin{aligned}
G_{n}^{*}\left(e_{2} ; x\right) \leq & x\left(x+2 b_{n} \beta\right)(1+n \beta) \\
& +x \frac{b_{n}}{n}(n \beta)^{2}(1+n \beta)+x\left(x+2 b_{n} \beta\right) n \beta \\
& +x \frac{b_{n}}{n}(n \beta)^{3}+x \frac{b_{n}}{n} .
\end{aligned}
$$

So, we obtain the desired results of Lemma 2.1. 
Now, in order to get an approximation result, we consider $\beta$ as a sequence of positive real numbers such that $\beta=\beta_{n}$ and $\lim _{n \rightarrow \infty} n \beta_{n}=0$.

We show here that the operators $G_{n}^{*}(f)$ satisfy the required conditions for the weighted Korovkin type theorem.

Theorem 2.4. Suppose that $n \in \mathbb{N},\left(b_{n}\right)$ and $\left(\beta_{n}\right)$ are sequence of positive numbers such that $\lim _{n \rightarrow \infty} b_{n}=\infty, \lim _{n \rightarrow \infty} b_{n} / n=0$ and $\lim _{n \rightarrow \infty} n \beta_{n}=0$. Then for each $f \in C_{\rho}^{k}\left(\mathbb{R}^{+}\right)$, we have

$$
\lim _{n \rightarrow \infty}\left\|G_{n}^{*} f-f\right\|_{\rho}=0 .
$$

Proof. By Lemma 2.2, $\left(G_{n}^{*}\right)$ are linear operators acting from $C_{\rho}\left(\mathbb{R}^{+}\right)$to $B_{\rho}\left(\mathbb{R}^{+}\right)$. Indeed, from (2.5) and (2.22), we easily obtain that

$$
\left|G_{n}^{*}(\rho ; x)\right| \leq\left(1+x^{2}\right) K_{n} .
$$

On the other hand, using $(2.5),(2.11)$ and $(2.22)$, one can write

$$
\begin{gathered}
\left\|G_{n}^{*}\left(e_{0}\right)-1\right\|_{\rho}=0, \\
\left\|G_{n}^{*}\left(e_{1}\right)-x\right\|_{\rho}=0,
\end{gathered}
$$

and

$$
\begin{aligned}
\left\|G_{n}^{*}\left(e_{2}\right)-x^{2}\right\|_{\rho} \leq & \sup \left\{\left|\frac{x^{2}}{1+x^{2}}\left(2 n \beta_{n}\right)\right|\right. \\
& +\left|\frac{2 x}{1+x^{2}} b_{n} \beta_{n}\left(1+n \beta_{n}\right)\right| \\
& \left.+\frac{x}{1+x^{2}} \frac{b_{n}}{n}\left(n \beta_{n}\right)^{2}\left(1+2 n \beta_{n}\right)+\frac{x}{1+x^{2}} \frac{b_{n}}{n}\right\},
\end{aligned}
$$

by the hypothesis, we have

$$
\lim _{n \rightarrow \infty}\left\|G_{n}^{*}\left(e_{2}\right)-x^{2}\right\|_{\rho}=0
$$

So the proof is completed.

In the following theorem, we give approximation results of the operators $\left(G_{n}^{*}\right)$ with the help of universal Korovkin-type theorem.

Theorem 2.5. Let $\left(G_{n}^{*}\right)$ be the operators given by (1.5). Then, for any $f \in C[0, \infty) \cap E$, the following relation holds

$$
\lim _{n \rightarrow \infty} G_{n}^{*}(f ; x)=f(x)
$$

uniformly on each compact subset of $[0, \infty)$, where

$$
E:=\left\{f: x \in[0, \infty), \frac{f(x)}{1+x^{2}} \text { is convergent as } x \rightarrow \infty\right\} .
$$

Proof. Using universal Korovkin-type theorem [5], it is sufficient to prove that the operators $\left(G_{n}^{*}\right)$ verify the conditions

$$
\lim _{n \rightarrow \infty} G_{n}^{*}\left(e_{i} ; x\right)=x^{i}, i=0,1,2,
$$

uniformly on each compact subset of $[0, \infty)$. From $(2.2)-(2.4)$, we obtain that the mentioned conditions are supplied. So the proof is completed. 


\section{Rate of convergence}

In this section, we compute rate of convergence of the operators $\left(G_{n}^{*}\right)$ by means of the modulus of continuity.

Let $f \in \tilde{C}[0, \infty)$ such that $f$ is uniformly continuous for any $\delta>0$. The usual modulus of continuity for $f$ is defined as

$$
\omega(f, \delta)=\sup _{\substack{|x-y| \leq \delta \\ x, y \in[0, \infty)}}|f(x)-f(y)| .
$$

For $f \in \tilde{C}[0, \infty)$ and any $x, y \in[0, \infty)$, we have

$$
|f(x)-f(y)| \leq \omega(f ;|y-x|),
$$

and for any $\delta>0$

$$
|f(x)-f(y)| \leq \omega(f, \delta)\left(\frac{|x-y|}{\delta}+1\right) .
$$

Now, we give the following result.

Theorem 3.1. Suppose that $n \in \mathbb{N},\left(b_{n}\right)$ and $\left(\beta_{n}\right)$ are sequences of positive numbers such that $\lim _{n \rightarrow \infty} b_{n}=\infty, \lim _{n \rightarrow \infty} b_{n} / n=0$ and $\lim _{n \rightarrow \infty} n \beta_{n}=0$. Then for each $f \in$ $C[0, \infty) \cap E$, we have

$$
\left|G_{n}^{*}(f ; x)-f(x)\right| \leq 2 \omega\left(f ; \delta_{n}(x)\right),
$$

where $\delta_{n}(x)=x^{2}\left(2 n \beta_{n}\right)+2 x b_{n} \beta_{n}\left(1+n \beta_{n}\right)+x \frac{b_{n}}{n}\left(n \beta_{n}\right)^{2}\left(1+2 n \beta_{n}\right)+x \frac{b_{n}}{n}$.

Proof. Using the linearity and positivity of $G_{n}^{*}(f ; x)$ and then applying (3.1), we have

$$
\begin{aligned}
\left|G_{n}^{*}(f ; x)-f(x)\right| \leq & G_{n}^{*}(|f(t)-f(x)| ; x) \\
\leq & (1+n \beta)^{1-n} \sum_{k=0}^{n} \omega(f, \delta)\left(1+\frac{|t-x|}{\delta}\right)\left(\begin{array}{l}
n \\
k
\end{array}\right) \frac{x}{b_{n}}\left(\frac{x}{b_{n}}+k \beta\right)^{k-1} \\
& \times\left(1-\frac{x}{b_{n}}\right)\left[1-\frac{x}{b_{n}}+(n-k) \beta\right]^{n-k-1} \\
= & \omega(f, \delta)(1+n \beta)^{1-n} \sum_{k=0}^{n}\left(1+\frac{|t-x|}{\delta}\right)\left(\begin{array}{l}
n \\
k
\end{array}\right) \frac{x}{b_{n}}\left(\frac{x}{b_{n}}+k \beta\right)^{k-1} \\
& \times\left(1-\frac{x}{b_{n}}\right)\left[1-\frac{x}{b_{n}}+(n-k) \beta\right]^{n-k-1},
\end{aligned}
$$

from $(2.2)$, one has

$$
\begin{aligned}
\left|G_{n}^{*}(f ; x)-f(x)\right| \leq & \omega(f, \delta)\left\{1+\frac{1}{\delta}\left(1+n \beta_{n}\right)^{1-n} \sum_{k=0}^{n}|t-x|\left(\begin{array}{l}
n \\
k
\end{array}\right) \frac{x}{b_{n}}\left(\frac{x}{b_{n}}+k \beta\right)^{k-1}\right. \\
& \left.\times\left(1-\frac{x}{b_{n}}\right)\left[1-\frac{x}{b_{n}}+(n-k) \beta\right]^{n-k-1}\right\} .
\end{aligned}
$$

By the Cauchy-Schwarz inequality, we reach to

$$
\left|G_{n}^{*}(f ; x)-f(x)\right| \leq \omega(f, \delta)\left\{1+\frac{1}{\delta}\left\{G_{n}^{*}\left((t-x)^{2} ; x\right)\right\}^{\frac{1}{2}}\right\} .
$$

On the other hand, using $(2.2),(2.3)$ and $(2.4)$, we get

$$
\begin{aligned}
G_{n}^{*}(\varphi ; x) & =G_{n}^{*}\left(e_{2} ; x\right)-2 x G_{n}^{*}\left(e_{1} ; x\right)+x^{2} G_{n}^{*}\left(e_{0} ; x\right) \\
& \leq x^{2}\left(2 n \beta_{n}\right)+2 x b_{n} \beta_{n}\left(1+n \beta_{n}\right)+x \frac{b_{n}}{n}\left(n \beta_{n}\right)^{2}\left(1+2 n \beta_{n}\right)+x \frac{b_{n}}{n} .
\end{aligned}
$$


Let choose $\delta=\delta_{n}(x):=x^{2}\left(2 n \beta_{n}\right)+2 x b_{n} \beta_{n}\left(1+n \beta_{n}\right)+x \frac{b_{n}}{n}\left(n \beta_{n}\right)^{2}\left(1+2 n \beta_{n}\right)+x \frac{b_{n}}{n}$, then we have

$$
\left|G_{n}^{*}(f ; x)-f(x)\right| \leq 2 \omega\left(f, \sqrt{\delta_{n}(x)}\right) .
$$

This concludes the proof.

\section{A-Statistical approximation by $\left(G_{n}^{*}\right)$}

In this section, we obtain $A$-statistical convergence of the Cheney-Sharma-Chlodovsky operators to identity operator on the weighted spaces. Let us recall the weighted Korovkin type approximation theorem for the A-statistical convergence was given by Duman and Orhan in [17].

Theorem 4.1. Let $A=\left(a_{n k}\right)$ be a nonnegative regular summability matrix and let $\bar{\rho}_{1, \bar{\rho}_{2}}$ weight functions such that $\overline{\rho_{1}}$

$$
\lim _{|x| \rightarrow \infty} \frac{\overline{\rho_{1}}(x)}{\overline{\rho_{2}}(x)}=0 .
$$

Assume that $\left(T_{n}\right)_{n \geq 1}$ is a sequence of positive linear operators from $C_{\overline{\rho_{1}}}(\mathbb{R})$ into $B_{\overline{\rho_{2}}}(\mathbb{R})$. One has

$$
s t_{A}-\lim _{n}\left\|T_{n} f-f\right\|_{\overline{\rho_{2}}}=0,
$$

for all $f \in C_{\overline{\rho_{1}}}(\mathbb{R})$ if and only if

$$
s t_{A}-\lim _{n}\left\|T_{n} F_{v}-F_{v}\right\|_{\overline{\rho_{1}}}=0, \quad k=0,1,2,
$$

where

$$
F_{v}(x)=\frac{x^{v} \overline{\rho_{1}}(x)}{1+x^{2}}, \quad v=0,1,2 .
$$

Corollary 4.2. [17]. Let $A=\left(a_{n k}\right)$ be a nonnegative regular summability matrix and let $\left(L_{n}\right)$ be a sequence of positive linear operators acting from $C_{\rho_{0}}\left(R^{+}\right)$into $B_{\rho_{\lambda}}\left(R^{+}\right), \lambda>0$ one has

if and only if

$$
s t_{A}-\lim _{n}\left\|L_{n} f-f\right\|_{\rho_{\lambda}}=0, \quad f \in C_{\rho_{0}}\left(R^{+}\right),
$$

$$
s t_{A}-\lim _{n}\left\|L_{n} e_{i}-e_{i}\right\|_{\rho_{0}}=0, i=0,1,2,
$$

where $\rho_{0}(x)=1+x^{2}$ and $\rho_{\lambda}(x)=1+x^{2+\lambda}, \lambda>0$.

Using this theorem the following Korovkin type statistical theorem can be proved for $\left(G_{n}^{*}\right)$ :

Theorem 4.3. Let $A=\left(a_{n k}\right)$ be a nonnegative regular summability matrix and let $\left(b_{n}\right)$ and $\left(\beta_{n}\right)$ be sequences of positive numbers such that st ${ }_{A}-\lim _{n \rightarrow \infty} b_{n} / n=0$, st $_{A}-\lim _{n \rightarrow \infty} n \beta_{n}=0$. Then for each $f \in C_{\rho}^{k}\left(\mathbb{R}^{+}\right)$, we have

$$
s t_{A}-\lim _{n \rightarrow \infty}\left\|G_{n}^{*} f-f\right\|_{\rho_{\lambda}}=0,
$$

where $\rho_{0}(x)=1+x^{2}$ and $\rho_{\lambda}(x)=1+x^{2+\lambda}, \lambda>0$.

Proof. Using Corollary 4.2, it is sufficient to prove that the operators $\left(G_{n}^{*}\right)$ verify the conditions given in (4.1). Indeed, from (2.2) and (2.3), it is clear that

$$
s t_{A}-\lim _{n}\left\|G_{n}^{*}\left(e_{0} ; .\right)-e_{0}\right\|_{\rho_{0}}=0
$$

and

$$
s t_{A}-\lim _{n}\left\|G_{n}^{*}\left(e_{1} ; .\right)-e_{1}\right\|_{\rho_{0}}=0 .
$$


Now using (2.4), one can have

which implies

$$
\begin{aligned}
\left\|G_{n}^{*}\left(e_{2}\right)-x^{2}\right\|_{\rho_{0}} \leq & \sup \left\{\left|\frac{x^{2}}{1+x^{2}}\left(2 n \beta_{n}\right)\right|\right. \\
& +\left|\frac{2 x}{1+x^{2}} b_{n} \beta_{n}\left(1+n \beta_{n}\right)\right| \\
& \left.+\frac{x}{1+x^{2}} \frac{b_{n}}{n}\left(n \beta_{n}\right)^{2}\left(1+2 n \beta_{n}\right)+\frac{x}{1+x^{2}} \frac{b_{n}}{n}\right\},
\end{aligned}
$$

$$
\begin{aligned}
\left\|G_{n}^{*}\left(e_{2}\right)-x^{2}\right\|_{\rho_{0}} & \leq 2 n \beta_{n}+2 b_{n} \beta_{n}\left(1+n \beta_{n}\right)+\frac{b_{n}}{n}\left(n \beta_{n}\right)^{2}\left(1+2 n \beta_{n}\right)+\frac{b_{n}}{n} \\
& =K_{n} .
\end{aligned}
$$

Now, for a given $\epsilon>0$, let us define the following sets:

$$
\begin{gathered}
M:=\left\{k:\left\|G_{n}^{*}\left(e_{2} ; .\right)-e_{2}\right\|_{\rho_{0}} \geq \epsilon\right\}, \\
M_{1}:=\left\{k: 2 n \beta_{n} \geq \frac{\epsilon}{5}\right\}, \\
M_{2}:=\left\{k: 2 b_{n} \beta_{n}\left(1+n \beta_{n}\right) \geq \frac{\epsilon}{5}\right\}, \\
M_{3}:=\left\{\frac{1}{n}(n \beta)^{2}(1+2 n \beta) \geq \frac{\epsilon}{5}\right\}, \\
M_{4}:=\left\{\frac{b_{n}}{n}\left(n \beta_{n}\right)^{2}\left(1+2 n \beta_{n}\right) \geq \frac{\epsilon}{5}\right\}, \\
M_{5}:=\left\{\frac{b_{n}}{n} \geq \frac{\epsilon}{5}\right\} .
\end{gathered}
$$

Then, we see that $M \subseteq M_{1} \cup M_{2} \cup M_{3} \cup M_{4} \cup M_{5}$. Therefore, we get

$$
\sum_{n:\left\|G_{n}^{*}\left(e_{2} ; .\right)-e_{2}\right\|_{\rho_{0}} \geq \epsilon} a_{k, n} \leq \sum_{n \in M_{1}} a_{k, n}+\sum_{n \in M_{2}} a_{k, n}+\sum_{n \in M_{3}} a_{k, n}+\sum_{n \in M_{4}} a_{k, n}+\sum_{n \in M_{5}} a_{k, n}
$$

and, taking the limit $k \rightarrow \infty$ in (4.3), we have

$$
s t_{A}-\lim _{n}\left\|G_{n}^{*}\left(e_{2} ; .\right)-e_{2}\right\|_{\rho_{0}}=0 .
$$

This proves the theorem.

Remark 4.4. In Theorem 4.3, we have stronger results than approximation theorems given in section 2 . Indeed, Theorem 4.3 may be useful when $n \beta_{n}$ and $b_{n} / n$ does not convergent to zero as $n \rightarrow \infty$.

The following example shows that there exist some sequences $\left(\beta_{n}\right)$ and $\left(b_{n}\right)$ such that $A$-satistical convergence holds but ordinary convergence does not hold for $n \beta_{n}$ and $b_{n} / n$.

Example 4.5. Let $\left(b_{n}\right)$ and $\left(\beta_{n}\right)$ be the sequences defined by

$$
b_{n}=\left\{\begin{array}{lc}
e^{n^{2}}, & \text { if } n \text { is a perfect square } \\
\sqrt{n}, & \text { otherwise. }
\end{array}\right.
$$

and

$$
\beta_{n}=\left\{\begin{array}{lc}
n, & \text { if } n \text { is a perfect square } \\
\frac{1}{n^{2}}, & \text { otherwise. }
\end{array}\right.
$$

It is easy to see that $b_{n} / n$ and $n \beta_{n}$ are not convergent but statistically convergent, i.e., $C_{1}$-statistically convergent. 


\section{References}

[1] A.M. Acu, C.V.Muraru, D.F. Sofonea and A.V. Radu, Some approximation properties of a Durrmeyer variant of q-Bernstein-Schurer operators, Math. Methods Appl. Sci. 39 (18), 5636-5650, 2016.

[2] O. Agratini and O. Doğru, Weighted Approximation by q-szász King type operators, Taiwanese J. Math. 14 (4), 1283-1296, 2010.

[3] O. Agratini and I.A. Rus, Iterates of a class of dicsrete linear operators via contraction principle, Comment. Math. Univ. Carolin. 44 (3), 555-563, 2003.

[4] P.N. Agrawal, V. Gupta, A.S. Kumar and A. Kajla. Generalized Baskakov-Szász type operators, Appl. Math. Comput. 236, 311-324, 2014.

[5] F. Altomare and M. Campiti, Korovkin-type approximaton theory and its applications, Walter de Gruyter, Berlin-New York, 1994.

[6] G. Başcanbaz-Tunca and F. Taşdelen, On Chlodovsky form of the Meyer-König and Zeller operators, An. Univ. Vest Timiş. Ser. Mat.-Inform. 49 (2), 137-144, 2011.

[7] G. Başcanbaz-Tunca, A. Erençin and F. Taşdelen, Some properties of Bernstein type Cheney and Sharma operators, Gen. Math. 24 (1-2), 17-25, 2016.

[8] G. Başcanbaz-Tunca, A. Erençin and H. G. İnce-İlarslan, Bivariate Cheney and Sharma Operators on Simplex, Hacet. J. Math. Stat. 47 (4), 793-804, 2018.

[9] J. Boos, Classical and Modern Methods in Summability, Oxford University Press, Oxford, 2000.

[10] R.C. Buck, The measure theoretic approach to density, Amer. J. Math. 68, 560-580, 1946.

[11] R.C. Buck, Generalized asymptotic density, Amer. J. Math. 75, 335-346, 1953.

[12] I. Büyükyazıcı, Approximation by Stancu-Chlodowsky polynomials, Comput. Math. Appl. 59, 274-282, 2010.

[13] E.W. Cheney and A. Sharma, On a generalization of Bernstein polynomials, Riv. Mat. Univ. Parma 2 (5), 77-84, 1964.

[14] I. Chlodovsky, Sur le development des fonctions $¥ ¥$ fines dans un interval infinien series de polyn omes de S.N. Bernstein, Compositio Math. 4, 380-392, 1937.

[15] M. Craciun, Approximation operators constructed by means of Sheffer sequences, Rev. Anal. Numér. Théor. Approx. 30 (2), 135-150, 2001.

[16] O. Duman, M.K. Khan, and C. Orhan, A-statistical convergence of approximating operators, Math. Inequal. Appl. 6 (4), 689-699, 2003.

[17] O. Duman and C. Orhan, Statistical approximation by positive linear operators, Studia Math. 161 (2), 187-197, 2004.

[18] H. Fast, Sur la convergence statistique, Colloq. Math. 2, 241-244, 1951.

[19] J.A. Fridy, On statistical convergence, Analysis, 5 (4), 301-313, 1985.

[20] J.A. Fridy and H.I. Miller, A matrix characterization of statistical convergence, Analysis 11, 59-66, 1991.

[21] A.D. Gadjiev, The convergence problem for a sequence of positive linear operators on unbounded sets and theorems analogues to that of P.P. Korovkin, Dokl. Akad. Nauk SSSR 218, 1974.

[22] A.D. Gadjiev, Theorems of the type of P. P. Korovkin's theorems (in Russian), Math. Z. 205, 781-786, 1976. Translated in Maths Notes, 20 (5-6), 995-998, 1977.

[23] A.D. Gadjiev and C. Orhan, Some approximation theorems via statistical convergence, Rocky Mountain J. Math. 32, 129-138, 2002.

[24] A.D. Gadjiev, R.O. Efendiev and E. Ibikli, Generalized Bernstein Chlodowsky polynomials, Rocky Mountain J. Math. 28 (4), 1267-1277, 1998.

[25] A. Izgi, Rate of approximation new-type generalization of Chlodovsky polynomial, Gen. Math. 21 (2), 125-140, 2013. 
[26] H. Karsli and E. Ibikli, Convergence rate of a new Bezier variant of Chlodowsky operators to bounded variation functions, J. Comput. Appl. Math. 212 (2), 431-443, 2008.

[27] H. Karsli and V. Gupta, Some approximation properties of q-Chlodowsky operators, Appl. Math. Comput. 195 (1), 220-229, 2008.

[28] A.S. Kumar and T. Acar, Approximation by generalized Baskakov-Durrmeyer-Stancu type operators, Rend. Circ. Mat. Palermo, 65 (3), 411-424, 2016.

[29] M. Mursaleen, K.J. Ansari and A. Khan, Some approximation results by $(p, q)$-analogue of Bernstein-Stancu operators, Appl. Math. Comput. 264, 392-402, 2015.

[30] M. Mursaleen, A.H. Al-Abieda and A.M. Acu, Approximation by Chlodowsky type of Szász operators based on Boas-Buck type polynomials, Turk. J. Math 42, 2243-2259, 2018.

[31] M.A. Özarslan, O. Duman, and H.M. Srivastava, Statistical approximation results for Kantorovich-type operators involving some special polynomials, Math. Comput. Model. 48 (3-4), 388-401, 2008.

[32] D. Söylemez and M. Unver, Korovkin Type Theorems for Cheney-Sharma Operators via Summability Methods, Results Math. 72, 1601-1612, 2017.

[33] M.W. Müller, Approximation by Cheney-Sharma-Kantorovic Polynomials in the $L_{p^{-}}$ Metric, Rocky Mountain J. Math. 19 (1), 281-291, 1989.

[34] M. Unver, Abel transform of positive linear operators, in: ICNAAM 2013, AIP Conference Proceedings 1558, 1148-1151, 2013.

[35] D.D. Stancu and C. Cismaşiu, On an approximating linear positive operator of Cheney-Sharma, Rev. Anal. Numèr. Thèor. Approx. 26 (1-2), 221-227, 1997.

[36] D.D. Stancu and E.I. Stoica, On the use Abel-Jensen type combinatorial formulas for construction and investigation of some algebraic polynomial operators of approximation, Stud. Univ. Babeş-Bolyai Math. 54 (4), 167-182, 2009. 